\title{
miRNA-10I-5p inhibits the growth and aggressiveness of NSCLC cells through targeting CXCL6
}

This article was published in the following Dove Press journal:

OncoTargets and Therapy

\section{Qi Chen ${ }^{1,2}$ \\ Dan $\mathrm{Liu}^{3}$ \\ Zhi Hu${ }^{4}$ \\ Cheng Luo' \\ Si Lin Zheng ${ }^{2}$}

'State Key Laboratory of Quality Research in Chinese Medicine, Macau Institute for Applied Research in Medicine and Health, Macau University of Science and Technology, Macau, China; ${ }^{2}$ Department of Nursing, The Affiliated Hospital of Southwest Medical University, Sichuan, 646000, China; ${ }^{3}$ Department of Respiratory and Critical Care Medicine, Pulmonary and Critical Care Medicine, The Affiliated Hospital of Southwest Medical University, Sichuan, 646000, China; ${ }^{4}$ Department of Thoracic Surgery, The Affiliated Hospital of Southwest Medical University, Sichuan, 646000, China
Correspondence: Si Lin Zheng Department of Nursing, The Affiliated Hospital of Southwest Medical University, No 25 Taiping Street, Luzhou, Sichuan, 646000, China

Email zhengsilinslin@foxmail.com
Background: The purpose of this study is to explore the potential biological roles of miR-101-5p in the progression of non-small-cell lung carcinoma (NSCLC).

Methods: The levels of miR-101-5p and chemokine (C-X-C motif) ligand 6 (CXCL6) in NSCLC tissues and cells were detected using the quantitative real-time PCR (qRT-PCR) assay. Proliferation, colony formation, migration and invasion assays were conducted using miR-101-5p-transfected NSCLC cells in vitro. The expression of CXCL6 was measured using immunofluorescence assay. Xenograft model and lung metastasis model were constructed to further reveal the precise roles of miR-101-5p in the lung metastasis and growth of NSCLC cells in vivo.

Results: miR-101-5p was underregulated in NSCLC tissues when compared with that in the normal controls. The levels of miR-101-5p were lower in NSCLC cells (H1975, A549, HCC827 and H1650) than in non-tumorigenic human bronchial epithelial cells (BEAS-2B). Overregulation of miR-101-5p restrained the aggressiveness phenotypes of NSCLC cells in vitro. Furthermore, overregulation of miR-101-5p reduced the tumor growth and pulmonary metastasis of NSCLC cells in vivo. CXCL6 was the target gene of miR-101-5p in NSCLC. The mRNA levels of CXCL6 were negatively associated with the levels of miR-101-5p in NSCLC tissues. Finally, the rescue experiments suggested that the inhibitory role of miR-101-5p was mediated by regulating the expression of CXCL6 in NSCLC.

Conclusion: These findings indicated that overregulation of miR-101-5p restrained the progression of NSCLC cells by targeting CXCL6 and might function as a potential therapeutic target for NSCLC.

Keywords: lung cancer, miR-101-5p, CXCL6, metastasis

\section{Introduction}

Human non-small-cell lung carcinoma (NSCLC) is still one of the leading causes for cancer-induced death worldwide. ${ }^{1}$ Radiotherapy and chemotherapy treatments remain the common curative therapy options for patients with NSCLC. Despite the great advance in the therapy options, including immunotherapy and targeted therapies, for this disease the clinical outcomes of patients with NSCLC are slightly improved because of recurrence and metastasis. ${ }^{2,3}$ Hence, it is very important to identify the molecular mechanisms behind the metastasis of NSCLC and explore the potential therapeutic targets for NSCLC.

miRNAs are a kind of small non-coding RNAs, which regulate the expression of target genes through promoting the degradation of target mRNA, via incompletely binding with the $3^{\prime}$-UTR of target proteins or inducing translational repression. ${ }^{4,5}$ 
Substantial evidence demonstrates that dysregulation of miRNAs is associated with the development and metastasis of several cancers, and miRNAs might be the potential treatment targets in cancers. ${ }^{6-9}$ For example, miR-661 serves as a suppressive miRNA in breast cancer and upregulation of miR-661 reduces the growth and invasion of cancer cells by regulating MTA1. ${ }^{10} \mathrm{miR}-490-3 \mathrm{p}$ regulates the proliferation and epithelial to mesenchymal transition (EMT) process of hepatocellular carcinoma (HCC) cells through regulating the expression of endoplasmic reticulum-Golgi intermediate compartment protein 3 (ERGIC3). ${ }^{11}$ Moreover, recent studies reported that miR-101 is significantly downregulated in NSCLC. ${ }^{12,13}$ miR-101-3p inhibits the growth and metastasis of NSCLC through blocking the phosphoinositide 3-kinase/protein kinase B (PI3K/AKT) signal pathway by targeting metastasis-associated lung adenocarcinoma transcript 1 (MALAT-1). ${ }^{14}$ Nevertheless, the potential roles of miR-101-5p in regulating the growth and metastasis of NSCLC cells are not yet well investigated.

Chemokines, which are a kind of small heparin-binding cytokines, regulate the migration of leukocytes through binding with G protein-coupled receptors (GPCR). ${ }^{15,16}$ More than 20 chemokine receptors and 50 chemokines have been found, which are divided into the following four kinds: $\mathrm{C}, \mathrm{CC}, \mathrm{CX} 3 \mathrm{C}$ and CXC. ${ }^{17,18}$ Chemokine receptor system, which extends to various types of neoplastic cells, is proved to be altered in the neoplastic tissues. ${ }^{19} \mathrm{C}-\mathrm{X}-\mathrm{C}$ motif chemokine ligand 6 (CXCL6) was first identified in osteosarcoma cell line, and it participates in the progression of various types of cancers, including prostate cancer, gastrointestinal tumors and breast cancer. ${ }^{20-23}$ In a murine model of melanoma, downregulation of CXCL6 inhibits the growth, invasion and metastasis of tumor cells. ${ }^{24}$ In addition, the angiogenic role of CXCL6 is associated with the intratumoral expression of matrix metallopeptidase 9 (MMP-9) and the degradation of the extracellular matrix (ECM). ${ }^{25}$ Previous investigations have demonstrated the important role of CXCL6/C-X-C motif chemokine receptor 6 (CXCL6/CXCR6) in the metastasis of different cancers, including colorectal cancer and NSCLC. ${ }^{20,23}$ All these results indicate that CXCL6/CXCR6 interaction is important for the metastasis and progression of cancer.

In this study, microarray analysis of the expression profiles using GEO databases demonstrated that the miR101-5p was significantly downregulated in NSCLC. Our subsequent experiments indicated that miR-101-5p inhibited the growth and metastatic abilities of NSCLC cells in vitro and in vivo. Further bioinformatics analysis and the luciferase reporter assay identified that CXCL6 was the direct target gene of miR-101-5p in NSCLC. Finally, we identified that miR-101-5p inhibited the growth and metastasis of NSCLC cell via targeting CXCL6.

\section{Materials and methods NSCLC tissues}

Fifty-five pairs of human NSCLC tissues and corresponding adjacent normal tissues were obtained from patients with NSCLC, who received surgery at the Affiliated Hospital of Southwest Medical University. No patients received treatment before clinical surgery. Ethical approval was obtained from the ethics committee of the Affiliated Hospital of Southwest Medical University. The study conforms to the Code of Ethics of the World Medical Association (Declaration of Helsinki) printed in the British Medical Journal (July 18, 1964). The clinicopathological characteristics of the mutations of patients with NSCLC are presented in Tables S1 and S2. Informed consent forms were signed by all patients before surgery. All tissues were conserved in liquid nitrogen for further RNA extraction.

\section{NSCLC cell lines and transfection}

Four NSCLC cell lines (A549, H1975, H1650 and HCC827), normal human fetal lung fibroblast (WI-38) and nontumorigenic human bronchial epithelial cell (BEAS-2B) were obtained from GuangZhou Jennio Biotech Co., Ltd (Guangzhou, Guangdong, China). 293 T cell line was purchased from Nanjing Cobioer Biotech Co., Ltd (Nanjing, Jiangsu, China). Cells were cultured in a humidified incubator at $37^{\circ} \mathrm{C}$ with $5 \% \mathrm{CO}_{2}$. miR-101-5p mimics, miRNA negative control (miR-NC), miR-101-5p inhibitor (miR-101-5 $\mathrm{p}^{\text {inhi }}$ ) and miRNA negative control inhibitor (miR-NC ${ }^{\text {inhi }}$ ) were obtained from Thermo Fisher Scientific (Waltham, MA). The small interfering RNA (siRNA) targeting CXCL6 (siCXCL6) and siRNA control (siCon) were bought from GenePharma (Shanghai, China). To increase the expression of CXCL6, CXCL6 cDNA was cloned into pcDNA3.1(+) vector (Genechem, Shanghai, China) and was transfected into NSCLC cells. An empty vector (EV) was used as control. miR-101-5p mimics or miR-101-5 $\mathrm{p}^{\text {inhi }}$ was transfected into cells using Lipofectamine ${ }^{\mathrm{TM}} 2000$ reagent (Thermo Fisher Scientific) according to manufacturer's protocol.

\section{Quantitative real-time PCR (qRT-PCR)}

RNA was extracted using TRIzol reagent (Thermo Fisher Scientific). RNA (1 $\mu \mathrm{g})$ was reverse transcribed into cDNA using the PrimeScript RT reagent kit (TakaraBio, Tokyo, Japan) 
and a TaqMan miRNA reverse transcription kit (Thermo Fisher Scientific). qRT-PCR was conducted using SYBR Premix Ex Taq $^{\mathrm{TM}}$ kit (TakaraBio) and miRNA-specific TaqMan miRNA assay kit (Thermo Fisher Scientific) in the Applied Biosystems 7500 Sequence Detection system (Thermo Fisher Scientific). The primers were as follows: miR-101-5p (forward primer: 5'-GCCGGCAGCATTATGTCAAT-3'; reverse primer: 5'-GCCAGCAGCTTGATGTCAAT-3'), CXCL6 (forward primer: 5'-AGAGCTGCGTTGCACTTGTT-3'; reverse primer: 5'-GCAGTTTACCAATCGTTTTGGGG-3'), U6 (forward primer: 5'-AAAGCAAATCATCGGACGACC-3'; reverse primer: 5'-GTACAACACATTGTTTCCTCGGA-3'), GAPDH (forward primer: 5'-TGTGGGCATCAA TGGATTTGG-3'; reverse primer: 5'-ACACCATGTAT TCCGGGTCAAT-3'), TEAD1 (forward primer: 5'-ATGGA AAGGATGAGTGACTCTGC-3'; reverse primer: 5'-TCCC ACATGGTGGATAGATAGC-3'), ZBTB18 (forward primer: 5'-TCTGAGCGAGCAGAGACAC-3'; reverse primer: 5'-GGTCCTTGTAAAAGAGGTGGAAA-3'), CCDC117 (forward primer: 5'-CGCGGACGTGTTTCTGTTC-3'; reverse primer: 5'-CCAGTCATTAGGACCAGCACA-3'), AIMP1 (forward primer: 5'-GGTACTCCACTGCACGCTAAT-3'; reverse primer: 5'-CCAGAAGATACGGTTGTTACTGC-3') and PPP2R5E (forward primer: 5'-TCAGCAC CAACTACTCCTCCA-3'; reverse primer: 5'-GCCTT GAGACCTAAACTGTGAG-3'). Candidate reference genes for normalization and the expression stability were calculated by the NormFinder program and are shown in Table S3. U6 and GAPDH were the internal controls. The comparative cycle threshold $(\mathrm{Ct})$ method was selected to detect the level by calculating using the $2^{(-\Delta \Delta \mathrm{Ct})}$ method.

\section{Cell counting kit-8 (CCK-8) assay}

NSCLC cell $\left(5 \times 10^{3}\right.$ cells/well) was cultured into 96-well plates. Then, CCK-8 solution (Beyotime, Shanghai, China) was added into the plate. After 2 hours, the OD value was detected at $450 \mathrm{~nm}$ using the Synergy ${ }^{\mathrm{TM}}$ HT Multi-Mode Microplate Reader (Bio-Tek, Winooski, VT, USA).

\section{Colony formation}

NSCLC cells $\left(1 \times 10^{3}\right.$ cells/well) were seeded into six-well plates and were cultured using complete medium for 4 weeks. Then, cell colonies were stained using $1 \%$ crystal violet, and the number of colonies was counted.

\section{Migration assay}

Cells were seeded into six-well plates to form confluence. After 24 hours, a wound was scratched using a $100 \mu \mathrm{L}$ pipette tip. Non-adherent cells were removed using fresh medium. Cells were cultured for 0 hour or 48 hours, and the wounds were photographed using the ZEN 2011 imaging software on a Zeiss invert microscope (Carl Zeiss, Hallbergmoos, Germany). ${ }^{26}$

\section{Invasion analysis}

The upper chamber of Transwell was pre-coated with Matrigel (BD Biosciences, San Jose, CA). A total of $1 \times 10^{5}$ cells were plated into the upper chamber of Transwell, and $600 \mu \mathrm{L}$ medium (containing 20\% FBS) was plated into the lower chamber. After 24 hours, the invaded cells were stained using $1 \%$ crystal violet. $^{27}$

\section{Immunofluorescence}

A549 cells were permeabilized using $0.1 \%$ Triton X-100 and were immunostained by incubating with antibody against CXCL6 (Boster Biotechnology, Nanjing, Jiangsu, China) overnight at $4^{\circ} \mathrm{C}$. Then, the cells were incubated with fluorescein isothiocyanate (FITC)-conjugated goat anti-rabbit secondary antibody (Boster Biotechnology). Nuclei were counterstained with DAPI (Boster Biotechnology). Images were taken and analyzed using the ZEN 2011 imaging software on a Zeiss invert microscope.

\section{In vivo nude mice tumorigenesis}

In order to generate miR-101-5p stable transfection cell line, A549 cells were transfected with miR-101-5p and were selected using $1 \mu \mathrm{g} / \mathrm{mL}$ puromycin (MedChemExpress, Monmouth Junction, NJ, USA). A total of $1 \times 10^{6} \mathrm{miR}-\mathrm{NC}$ or miR101-5p-transfected A549 cells were inoculated subcutaneously into $\mathrm{BALB} / \mathrm{c}$ nude mice ( $\mathrm{n}=6$ in each group). Tumor volume was detected every 3 days. After 3 weeks, all nude mice were sacrificed. In experimental metastasis assay, miR-NC or miR$101-5$ p-transfected A549 cells $\left(5 \times 10^{5}\right)$ were injected into nude mice via the lateral tail vein. After 4 weeks, mice were sacrificed, and the macroscopic metastases were examined using lung tissues. Animal experiments were approved by the Affiliated Hospital of Southwest Medical University. The animal experiment was conducted in accordance with the Institutional Guidelines and the Guide for the Care and Use of Laboratory Animals (NIH publication no 85-23, revised 1996).

\section{Luciferase reporter assay}

The 3 '-UTR of CXCL6 containing the binding sites within miR-101-5p was cloned into pGL3 luciferase reporter vector (Promega Corporation, Fitchburg, WI). The binding sites were mutated using the Quick-change site-directed 
mutagenesis kit (Agilent Technologies, Santa Clara, CA). The pGL3 luciferase reporter vector that contains the wild type (wt) 3'-UTR of CXCL6 or mutant type (mut) 3'-UTR of CXCL6 was transfected into 293 T cells in combination with miR-101-5p using Lipofectamine 2000 (Thermo Fisher Scientific). The luciferase activities were detected using the Dual-Luciferase Reporter Assay System (Promega Corporation), and the luciferase activity was normalized to Renilla activity. ${ }^{28}$

\section{Statistical analysis}

Data are presented as mean \pm SD and are analyzed using GraphPad Prism 5.0 (GraphPad Software, Inc., San Diego, CA). Statistical analysis was conducted using Student's $t$-test or one-way ANOVA followed by post hoc Dunnett's test. $P<0.05$ was considered as statistically significant.

\section{Results}

\section{miR-I0I-5p is downregulated in NSCLC}

The gene expression datasets that were used for statistical analysis were obtained from the GEO database with the accession codes GSE64591 and GSE53882. The screening was conducted using GEO datasets that contained lung cancer and adjacent normal tissues (Figure 1A). The 11 common potential downregulated miRNAs in lung cancer were screened out based on fold change $<-1$ as well as $P<0.05$ and are summarized in Figure 1B. Among these candidates, we focus on miR-101-5p, which was most significantly underregulated in NSCLC. The levels of miR-101-5p in 55 pairs of NSCLC tissues and corresponding normal tissues were evaluated using qRT-PCR assay. Our result suggested that miR-101-5p was markedly downregulated in NSCLC when compared with the corresponding normal lung samples (Figure 1B). Then, the levels of miR-101-5p in NSCLC cells (H1975, A549, HCC827 and H1650), non-tumorigenic human bronchial epithelial cell (BEAS-2B) and normal human fetal lung fibroblast (WI-38) were further explored using qRT-PCR assay. As expected, miR-101-5p was downregulated in NSCLC cells compared to that in two normal cell lines (Figure 1C). Thus, these results indicate that miR-101-5p is downregulated in NSCLC.
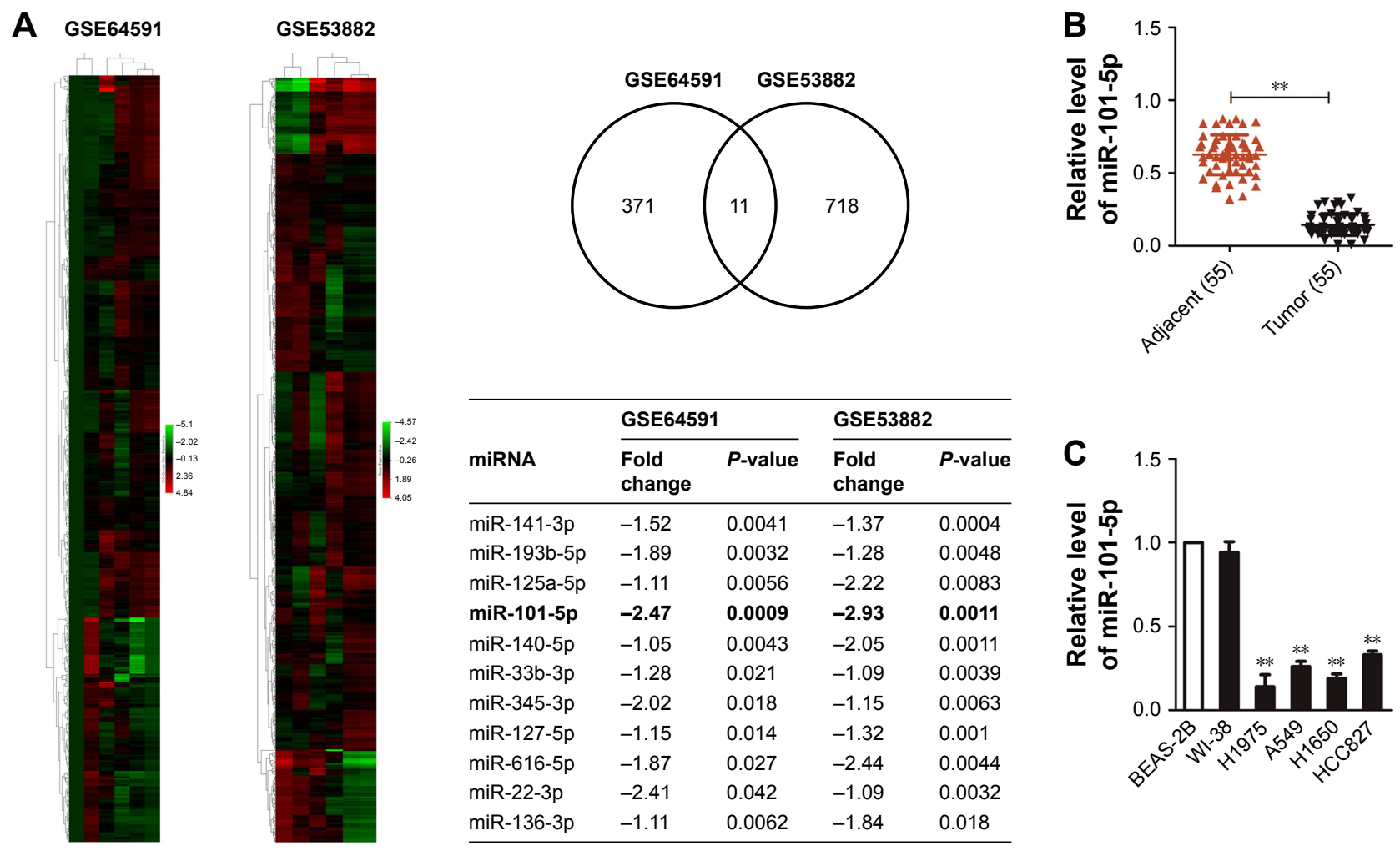

\begin{tabular}{|c|c|c|c|c|}
\hline \multirow[b]{2}{*}{ miRNA } & \multicolumn{2}{|c|}{ GSE64591 } & \multicolumn{2}{|c|}{ GSE53882 } \\
\hline & $\begin{array}{l}\text { Fold } \\
\text { change }\end{array}$ & $P$-value & $\begin{array}{l}\text { Fold } \\
\text { change }\end{array}$ & $P$-value \\
\hline miR-141-3p & -1.52 & 0.0041 & -1.37 & 0.0004 \\
\hline miR-193b-5p & -1.89 & 0.0032 & -1.28 & 0.0048 \\
\hline miR-125a-5p & -1.11 & 0.0056 & -2.22 & 0.0083 \\
\hline miR-101-5p & -2.47 & 0.0009 & -2.93 & 0.0011 \\
\hline miR-140-5p & -1.05 & 0.0043 & -2.05 & 0.0011 \\
\hline miR-33b-3p & -1.28 & 0.021 & -1.09 & 0.0039 \\
\hline miR-345-3p & -2.02 & 0.018 & -1.15 & 0.0063 \\
\hline miR-127-5p & -1.15 & 0.014 & -1.32 & 0.001 \\
\hline miR-616-5p & -1.87 & 0.027 & -2.44 & 0.0044 \\
\hline miR-22-3p & -2.41 & 0.042 & -1.09 & 0.0032 \\
\hline miR-136-3p & -1.11 & 0.0062 & -1.84 & 0.018 \\
\hline
\end{tabular}

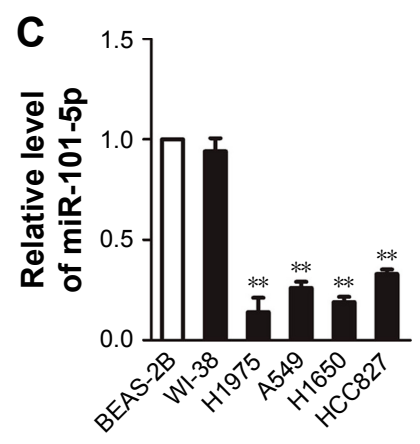

Figure I miR-10I-5p is downregulated in NSCLC.

Notes: (A) Expression profiling of dysregulated miRNAs in NSCLC tissues compared to normal tissues (NCBI/GEO/GSE6459I and NCBI/GEO/GSE53882). Summary of the common miRNAs in GSE6459I and GSE53882 (right panel). (B) The levels of miR-10I-5p were detected in 55 pairs of NSCLC tissues and tumor-adjacent tissues. **P $<0.05$ compared to normal tissues. (C) The levels of miR-I0I-5p in NSCLC cell lines (HI975, A549, HCC827 and HI650), WI-38 and BEAS-2B were detected using qRT-PCR assay. **P $<0.05$ compared to BEAS-2B cells.

Abbreviations: NSCLC, non-small-cell lung carcinoma; qRT-PCR, quantitative real-time PCR. 


\section{Upregulation of miR-I0I-5p inhibits the proliferation and aggressiveness of NSCLC cells}

A549 and H1975 cells were transfected with miR-NC or miR-101-5p, respectively, to reveal the precise role of miR-101-5p in the progression of NSCLC. The qRT-PCR assay suggested that miR-101-5p transfection increased the levels of miR-101-5p in A549 and H1975 cells (Figure 2A). Then, both CCK-8 and colony formation assay suggested that overregulation of miR-101-5p suppressed the proliferation of A549 and H1975 cells in vitro (Figure 2B and C). The wound healing assay indicated that upregulation of miR-101-5p markedly suppressed the migration of NSCLC cells in vitro (Figure 3A). Additionally, upregulation of miR-101-5p remarkably inhibited the invasion of A549 and H1975 cells (Figure 3B). Altogether, these findings suggest that overregulation of miR-101-5p suppresses the aggressiveness phenotypes of NSCLC cell in vitro.

\section{Upregulation of miR-I0I-5p reduces the growth of NSCLC cell in vivo}

The xenograft nude model using A549 cells that were transfected with miR-101-5p or miR-NC was constructed to reveal the role of miR-101-5p in the tumorigenicity of NSCLC cell in vivo. As shown in Figure 4A and B, the tumor volume and tumor weight of nude mice that were
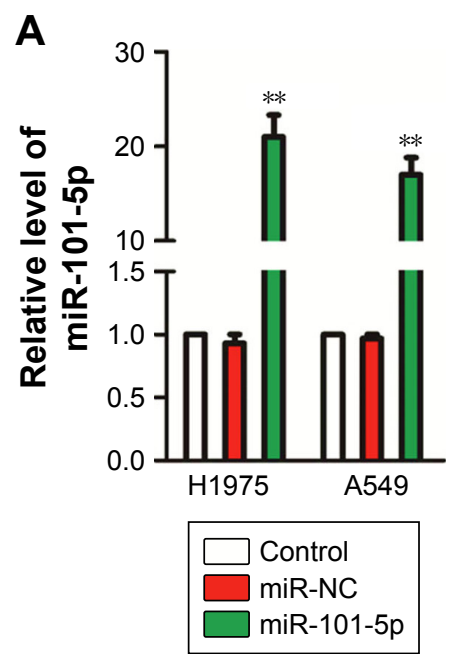

B

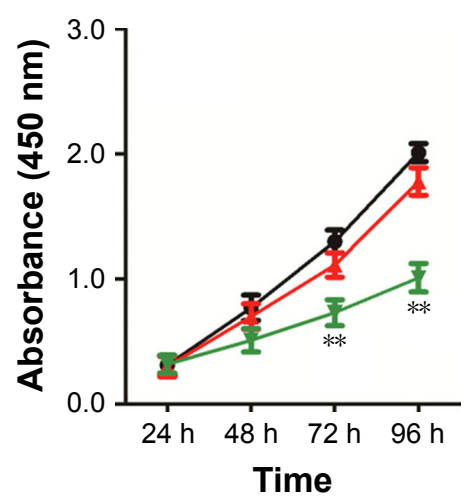

H1975

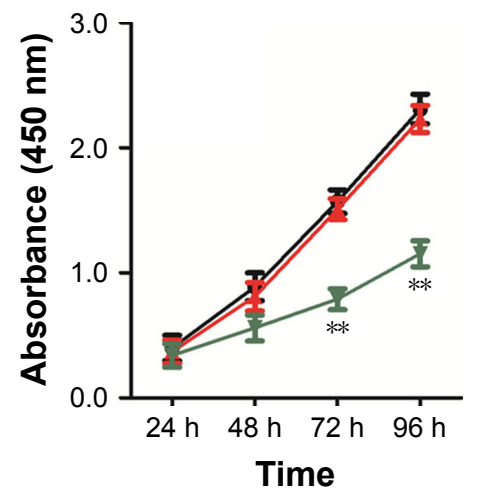

$\rightarrow$ Control $\leftarrow$ miR-NC $\rightarrow$ miR-101-5p

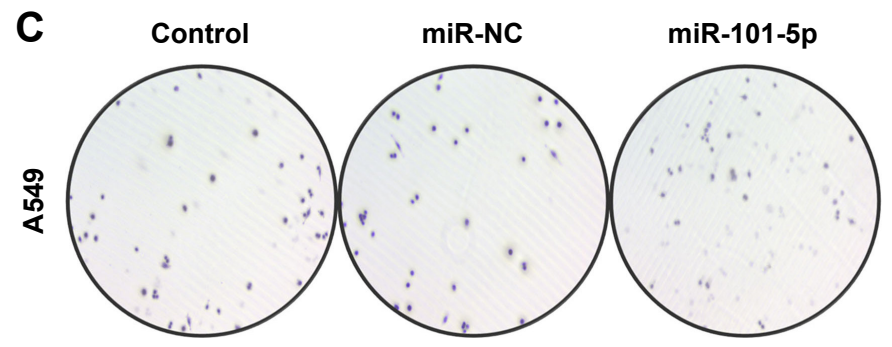

C

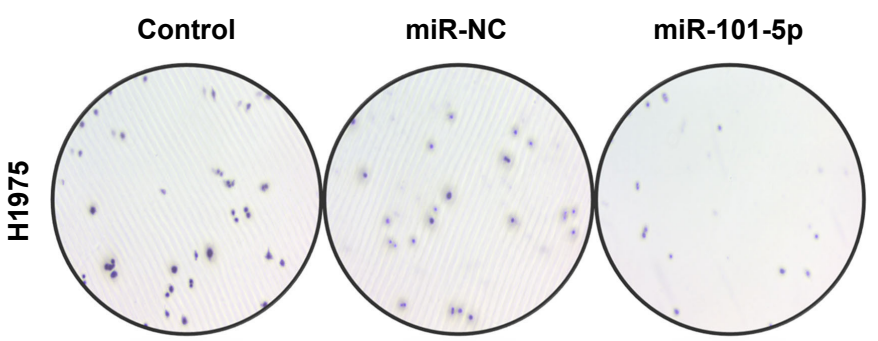

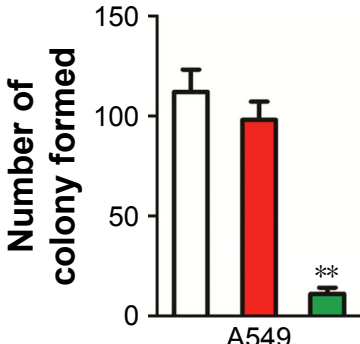
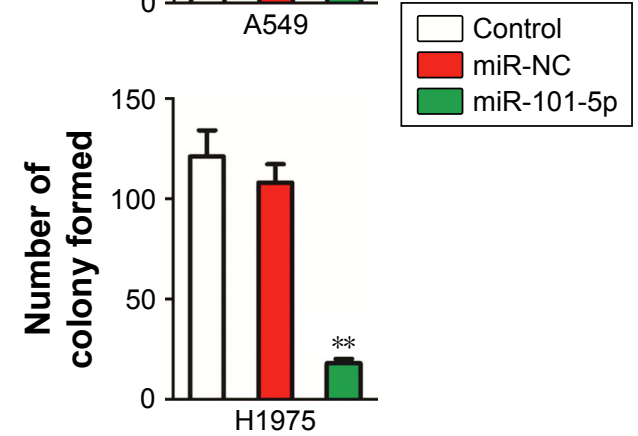

Figure 2 Upregulation of miR-I0I-5p inhibits the growth, migration and invasion of NSCLC cells.

Notes: (A) A549 and HI 975 cells were transfected with miR-I0I-5p or miRNA-NC, respectively. The level of miR-I0I-5p was detected using qRT-PCR. **P $<0.0$ I compared to control. (B) A549 and HI 975 cells were transfected with miR-I0I-5p or miRNA-NC. The proliferation of NSCLC cells was determined using CCK-8 assay. $* * P<0.0$ I compared to control. (C) A549 and HI 975 cells that were transfected with miR-I0I-5p or miRNA-NC was subjected to colony formation assay. $* * P<0.01$ compared to control. Abbreviations: CCK-8, cell counting kit-8; h, hours; NSCLC, non-small-cell lung carcinoma; qRT-PCR, quantitative real-time PCR. 
A

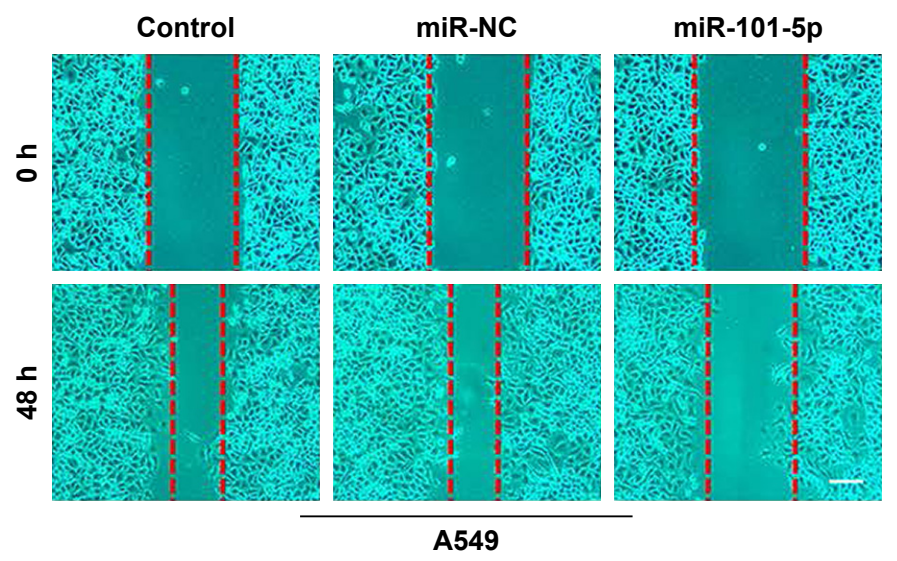

Control
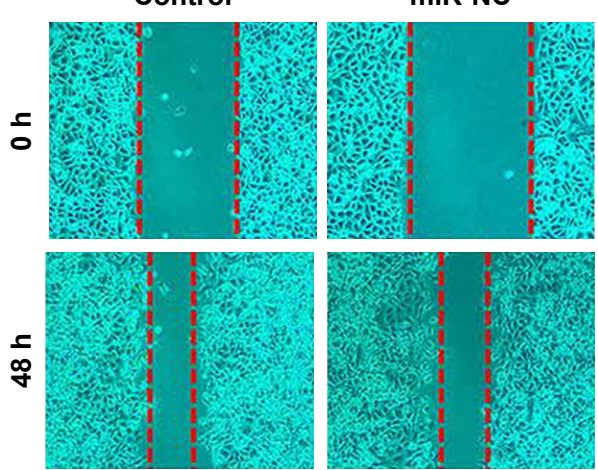

H1975

B

\section{๕ั)}

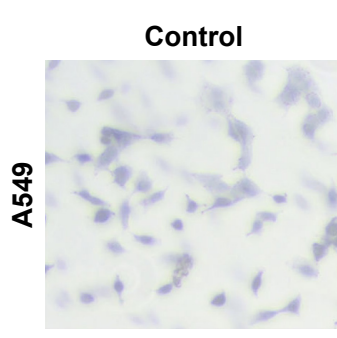

miR-NC

miR-101-5p
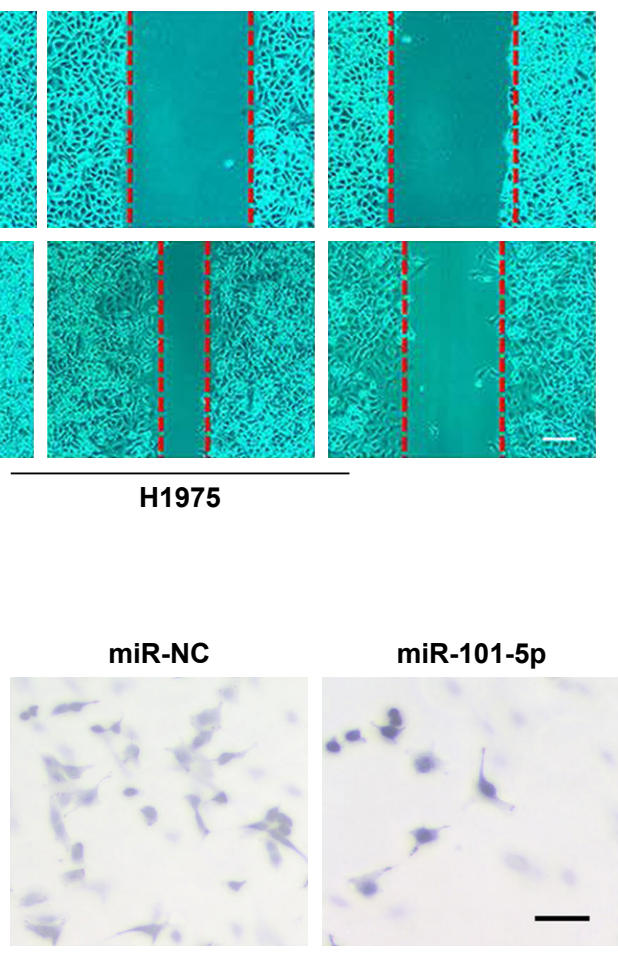

(1)
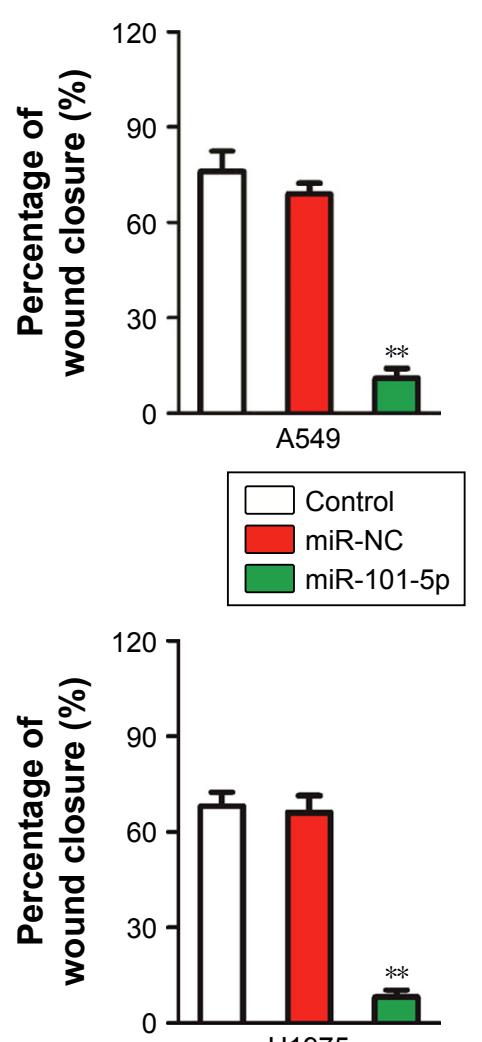
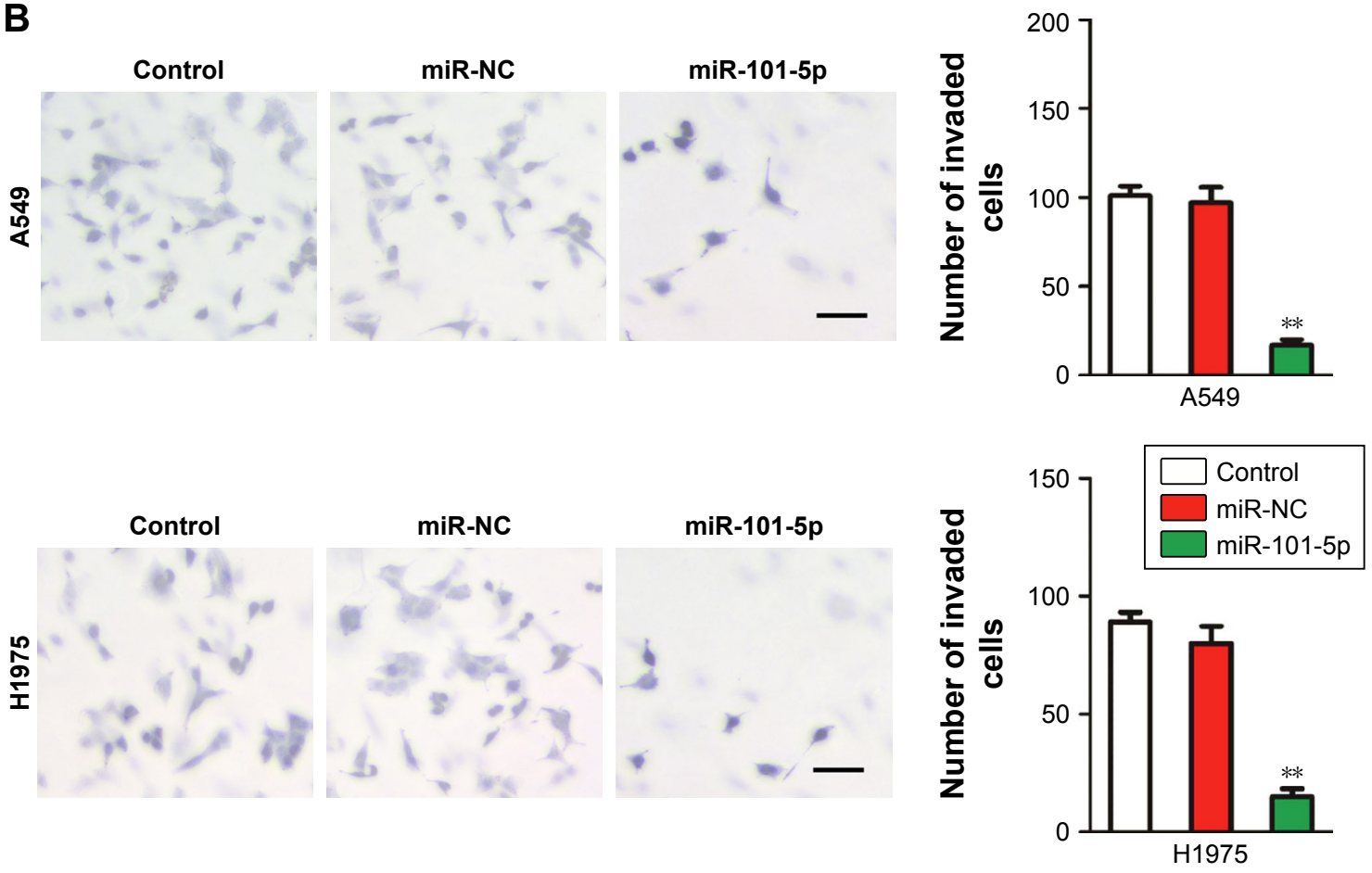

Figure 3 Upregulation of miR-10I-5p inhibits the migration and invasion of NSCLC cells.

Notes: (A) A549 and HI975 cells were transfected with miR-10I-5p or miR-NC, respectively. The migration of A549 and HI975 cells was detected using wound healing assay. ${ }^{* * P}<0.01$ compared to control. (B) A549 and HI975 cells were transfected with miR-10I-5p or miR-NC, respectively. The invasion of A549 and HI975 cells was detected using Transwell invasion assay. $* * P<0.01$ compared to control.

Abbreviations: h, hours; miR-NC, miRNA negative control; NSCLC, non-small-cell lung carcinoma. 
A

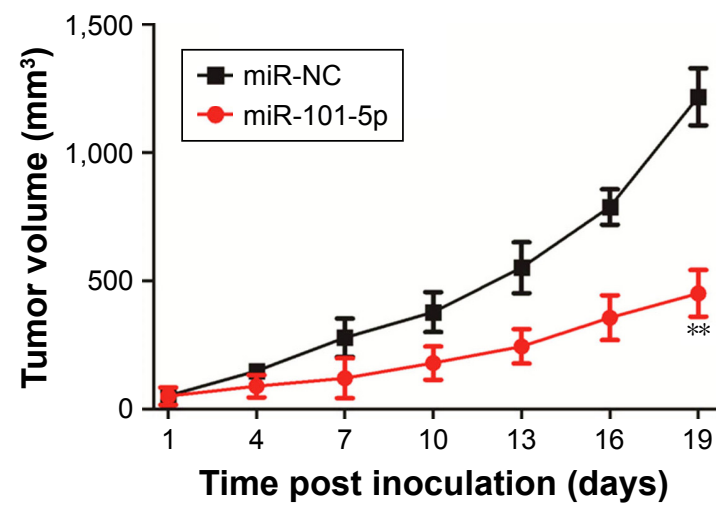

B

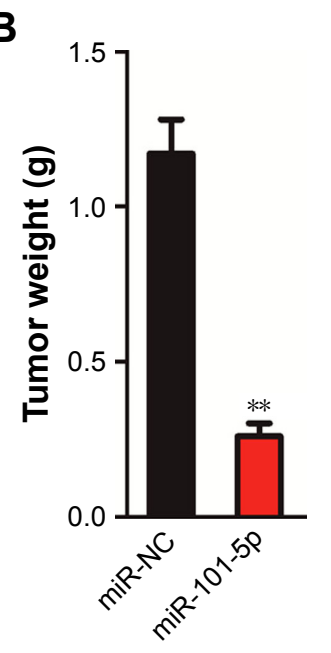

C

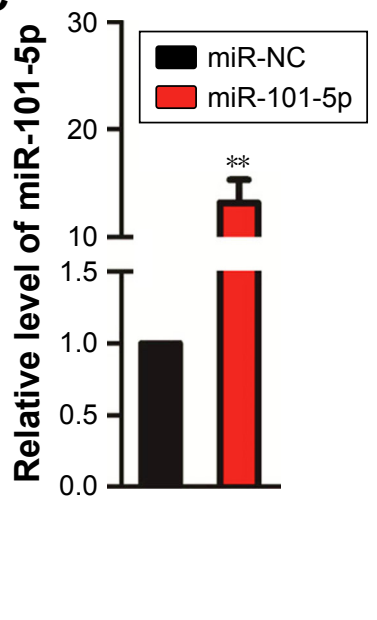

D

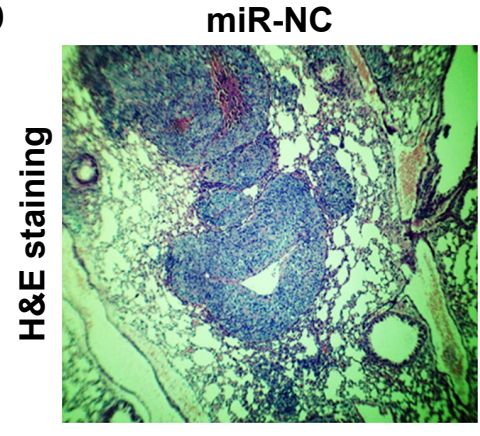

miR-101-5p

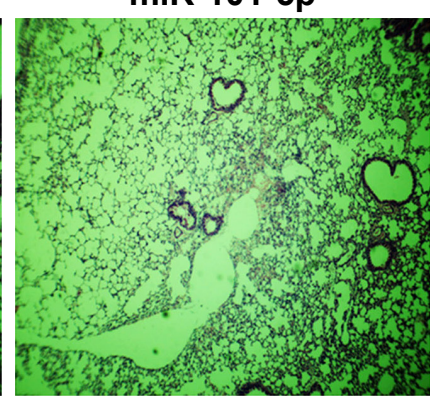

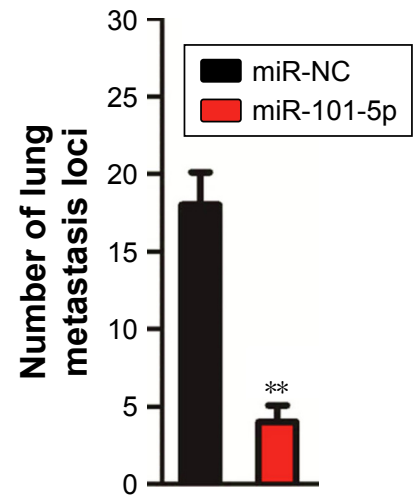

Figure 4 miR-101-5p inhibits the growth of A549 cells in vivo.

Notes: (A) A549 cells transfected with miR-NC or miR-10I-5p were implanted into nude mice via subcutaneous injection. Tumor growth curves revealed that NSCLC xenografts arising from the miR-10I-5p group grew slower than those arising from the control group. (B) Tumor weight. **P<0.0I compared to miR-NC. (C) The level of miR-10I-5p in tumor tissue was determined by qRT-PCR assay. (D) Representative picture of H\&E staining using lung tissue. The numbers of lung metastasis were quantified and showed by each data point, $* * P<0.01$ compared to miR-NC. $* * P<0.01$ compared to miR-NC.

Abbreviations: miR-NC, miRNA negative control; NSCLC, non-small-cell lung carcinoma; qRT-PCR, quantitative real-time PCR.

inoculated with miR-101-5p-transfected A549 cells were significantly decreased as compared with that in mice that were inoculated with miR-NC-transfected A549 cells. The levels of miR-101-5p in tumor tissues that were formed by miR-101-5p-transfected A549 cells were significantly higher than those in tumors that were formed by miRNC-transfected A549 cells (Figure 4C). An experimental metastasis model was applied to explore the impact of miR101-5p on the metastasis of NSCLC cells in vivo. miR-NC or miR-101-5p-transfected A549 cells were injected into nude mice via the lateral tail vein. Four weeks post cell inoculation, nude mice were sacrificed and lung tissues were checked. Injection of miR-NC-transfected A549 cells via lateral tail vein resulted in numerous lung metastasis, whereas injection of miR-101-5p-transfected A549 cells remarkably decreased the lung metastasis of A549 cells in vivo (Figure 4D). Collectively, these data reveal that miR-101-5p plays crucial roles in the growth and metastasis of NSCLC cell in vivo.

\section{CXCL6 is the direct target of miR-I0I-5p}

Then, the targets of miR-101-5p were predicted using online analysis tools, including TargetScan (http://www.targetscan. org), miRTarBase (http://mirtarbase.mbc.nctu.edu.tw/php/ index.php) and miRDB (http://www.mirdb.org/). ${ }^{29}$ The six common target genes that were obtained from three bioinformatics analysis tools are summarized in Figure 5A. In order to identify the direct gene of miR-101-5p, the mRNA levels of these genes in miR-101-5p- or miR-NCtransfected A549 cells were detected using qRT-PCR assay. As shown in Figure 5B, the mRNA level of CXCL6 was significantly inhibited by miR-101-5p in A549 cells. The 3'-UTR of CXCL6 contained the complementary binding sites within miR-101-5p as shown in Figure 5C. In addition, upregulation of $\mathrm{miR}-101-5 \mathrm{p}$ reduced the protein expression of CXCL6 in A549 and H1975 cells (Figure 5D). Notably, the luciferase activity of $293 \mathrm{~T}$ cells that were transfected with wt 3'-UTR of CXCL6 was reduced by miR-101-5p 
A
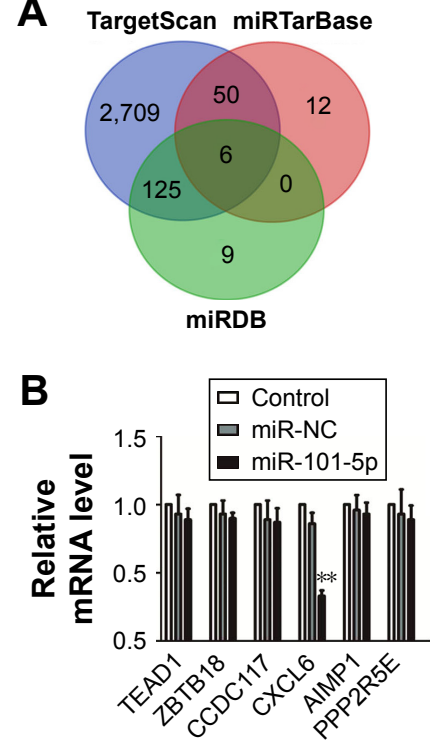

C

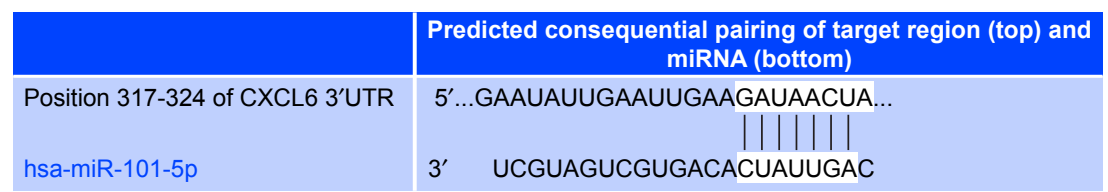

wt 3'-UTR of CXCL6 5'...GAAUAUUGAAUUGAAGAUAACUA...

\author{
||||||
}

miR-101-5p 3' UCGUAGUCGUGACACUAUUGAC

mut 3'UTR of CXCL6 5'...GAAUAUUGAAUUGAAGCGCUCUA...
D
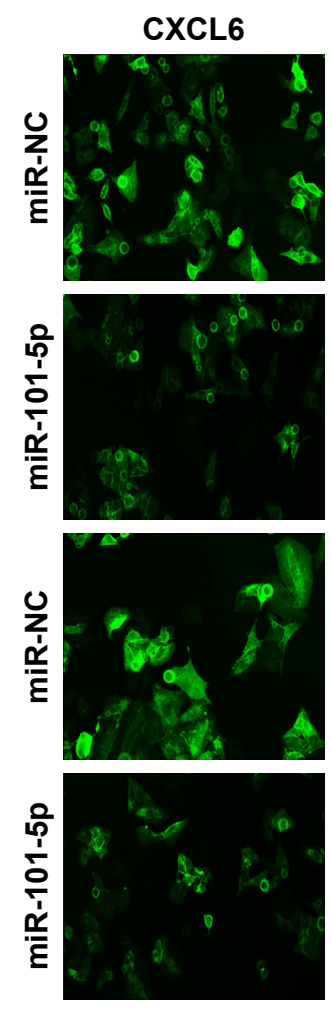

DAPI
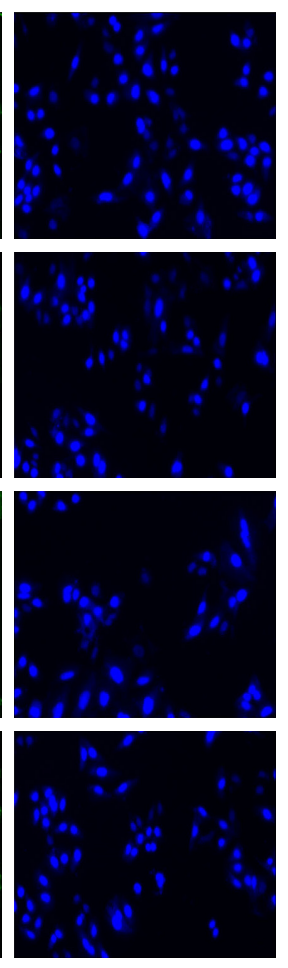

Merged
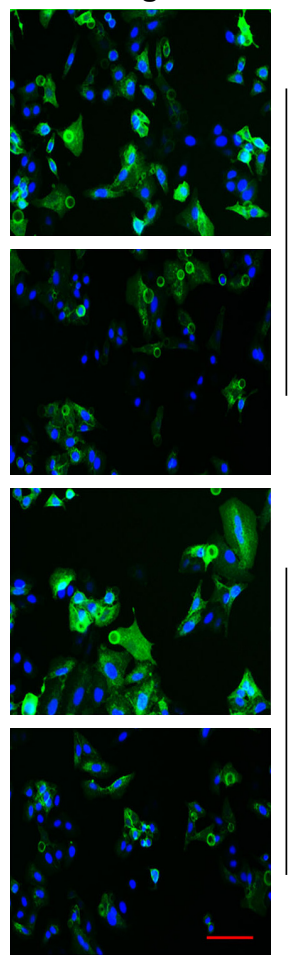

E

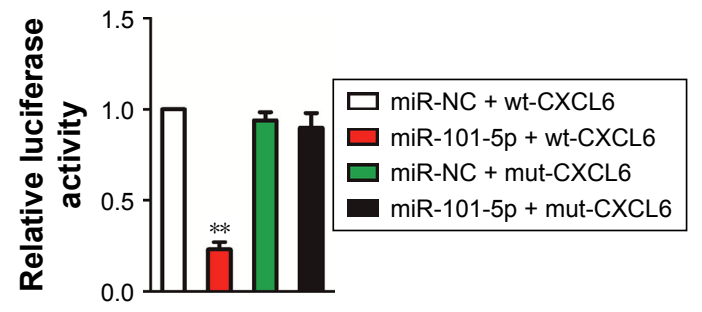

เ̛̣ $\mathbf{F}$

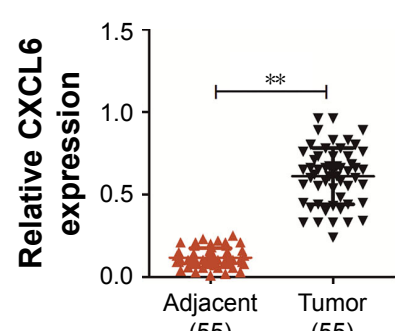

$\mathbf{G}$

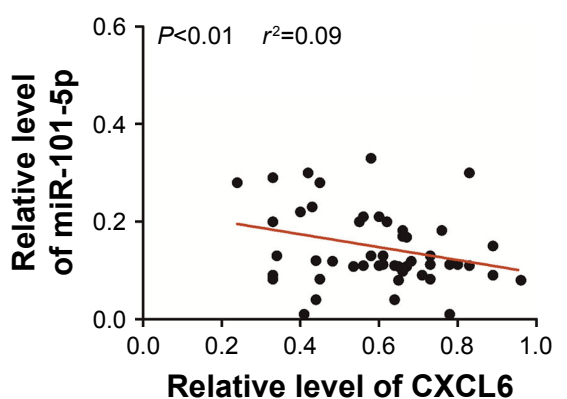

Figure 5 miR-10I-5p directly regulates the expression of CXCL6 in NSCLC cells.

Notes: (A) The complementary sequences of miR-10I-5p were discovered in 3'-UTR of CXCL6 mRNA using TargetScan, miRTarBase and miRDB. Venn graph represented the number of candidate common target genes determined by three bioinformatics analyses. (B) A549 cells were transfected with miR-NC or miR-I0I-5p, and the levels of potential target genes were measured by qRT-PCR assay. (C) The complementary sequences of miR-I0I-5P were discovered in 3 '-UTR of CXCL6 mRNA using TargetScan. The mutagenesis was performed in the complementary sites for the seed region of miR-10I-5p (wt, wild type; mut, mutant type). (D) A549 and HI975 cells were transfected with miR-10I-5p or miR-NC. Immunofluorescence assay indicated that upregulation of miR-I0I-5p reduced the expression of CXCL6. (E) miR-I0I-5p inversely modulated the luciferase activity of plasmids that contain wt $3^{\prime}-U T R$ of CXCL6. $* * P<0.01$. (F) The mRNA levels of CXCL6 were detected in NSCLC tissues compared to those in tumor-adjacent tissues. ${ }^{*} \mathrm{P}<0.0 \mathrm{I}$ compared to normal. (G) The levels of miR-I0I-5p were negatively correlated with the CXCL6 levels in NSCLC tissues.

Abbreviations: miR-NC, miRNA negative control; NSCLC, non-small-cell lung carcinoma; qRT-PCR, quantitative real-time PCR. 
(Figure 5E), whereas the luciferase activity of $293 \mathrm{~T}$ cells that were transfected with mut 3'-UTR of CXCL6 was not affected by miR-101-5p (Figure 5E). In addition, CXCL6 was markedly overregulated in NSCLC tissues than in the corresponding normal tissues (Figure 5F). Importantly, a negative correlation between the level of CXCL6 and miR101-5p level was found in NSCLC tissues (Figure 5G). All these findings suggest that miR-101-5p is the negative regulator of CXCL6 in NSCLC.

\section{CXCL6 is needed for miR-I0I-5p regulating the aggressiveness phenotypes of NSCLC cells}

Previous results demonstrated that CXCL6 is the target gene of miR-101-5p in NSCLC. Then, we performed the rescue experiments to further demonstrate that CXCL6 was needed for miR-101-5p regulating the aggressiveness phenotypes of NSCLC cells. A549 cells were transfected with miR-101-5p alone or cotransfected with miR-101-5p and pcDNA3.1(+) that contain CXCL6. The expression of CXCL6 was analyzed by immunofluorescence staining assay using anti-CXCL6 antibody (Figure 6A). Then, the proliferation, migration and invasion of A549 cells were investigated. As shown in Figure 6B and C, overexpression of CXCL6 increased the proliferation and colony formation of A549 cells that were inhibited by miR-101-5p transfection. Similarly, overexpression of CXCL6 significantly increased the migration and invasion abilities of A549 cells that were suppressed by miR-101-5p (Figure 6D and E). These findings indicate that miR-101-5p inhibits the growth, migration and invasion of NSCLC through targeting CXCL6.

\section{Confirmation that miR-I0I-5p inhibition of the aggressiveness phenotypes of NSCLC cells is dependent on CXCL6}

Finally, we conducted another rescue experiment to prove that miR-101-5p inhibition of the aggressiveness phenotypes of NSCLC cells was dependent on regulating CXCL6. A549 cells were transfected with miR-101-5 $\mathrm{p}^{\text {inhi }}$ alone or cotransfected with miR-101-5 $\mathrm{p}^{\text {inhi }}$ and siRNA targeting CXCL6. The protein expression of CXCL6 was assessed by immunofluorescence staining assay (Figure 7A). Then, the proliferation, migration and invasion abilities of NSCLC cells were investigated. As shown in Figure 7B and C, downexpression of CXCL6 obviously inhibited the proliferation and colony formation of A549 cells that were induced by miR-101-5 $\mathrm{p}^{\text {inhi }}$. Similarly, downexpression of CXCL6 dramatically suppressed the migration and invasion abilities of A549 cells that were promoted by miR-101-5p $\mathrm{p}^{\text {inhi }}$ (Figure 7D and E). These results indicate that $\mathrm{miR}-101-5 \mathrm{p}$ regulates the progression of NSCLC cells through targeting CXCL6.

\section{Discussion}

miRNAs have been proved to have close correlations with several tumors, including gastric cancer, breast carcinoma, colon cancer and ovarian cancer. ${ }^{30-33}$ Previous investigation indicates that miR-101 inhibits the growth and distant metastasis of lung cancer cells via regulating the expression of zinc finger E-box binding homeobox 1 (ZEB1). ${ }^{12}$ Among two mature miRNAs, miR-101-3p is generated from the $3^{\prime}$-ends of the precursor, and miR-101-5p is generated from the $5^{\prime}$-end of pre-miR-101. ${ }^{34}$ Accumulating evidence have demonstrated that the levels of miR-101-5p are decreased in many malignances, including liver cancer. ${ }^{35}$ In NSCLC, miR-101-3p suppresses the growth and metastasis of cancer cells via blocking the phosphoinositide 3-kinase/protein kinase $\mathrm{B}$ (PI3K/AKT) signaling pathway and regulating the expression of metastasis-associated lung adenocarcinoma transcript 1 (MALAT-1). ${ }^{14}$ Nevertheless, the level and precise role of miR-101-5p in NSCLC needed further investigation. In this work, we revealed that miR-101-5p was upregulated in NSCLC compared to that in the adjacent normal samples, which indicated that miR-101-5p might act as a tumor suppressor in the development of NSCLC. Consistently, miR-101-5p was significantly downregulated in NSCLC cells when compared to BEAS-2B or WI-38 cells. Then, we performed the gain- and loss-of-function experiments to reveal the roles of miR-101-5p in the growth, migration and metastasis of NSCLC cells. The results indicated that upregulation of miR-101-5p suppressed the proliferation, migration and invasion of NSCLC cells in vitro. Consistently, upregulation of miR-101-5p inhibited the metastasis and tumor growth of NSCLC cells in vivo.

We further investigated the potential targets of miR-101-5p using the public available database and demonstrated that CXCL6 gene was the target gene of miR-101-5p using the luciferase reporter analysis to explore the underlying mechanism of miR-101-5p as a suppressive miRNA in NSCLC. In addition, the expression of CXCL6 was inversely regulated by miR-101-5p in NSCLC cells, and the level of CXCL6 was negatively correlated with the level of miR-101-5p in NSCLC tissues.

Then, we proceeded to address the crucial role of miR-101-5p-CXCL6 axis in regulating aggressiveness 
A
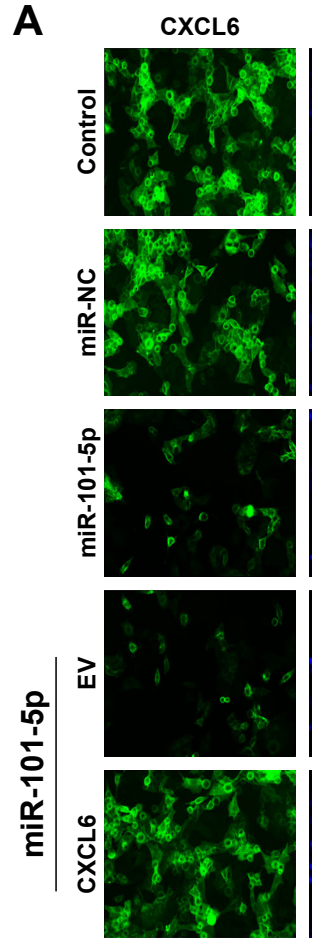
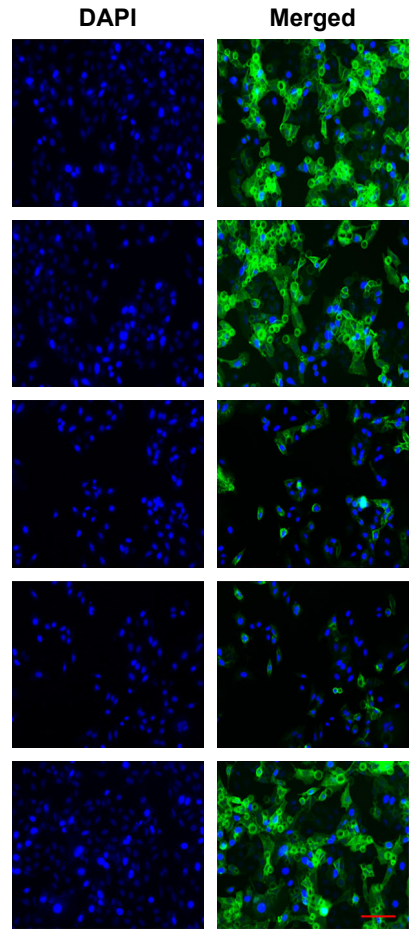

C

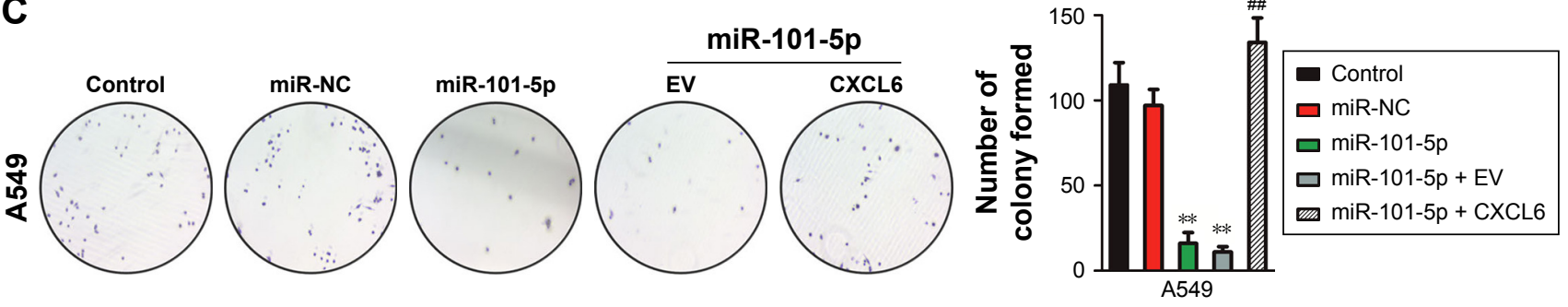

D

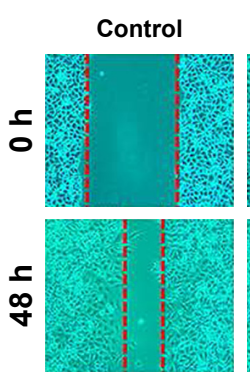

miR-NC
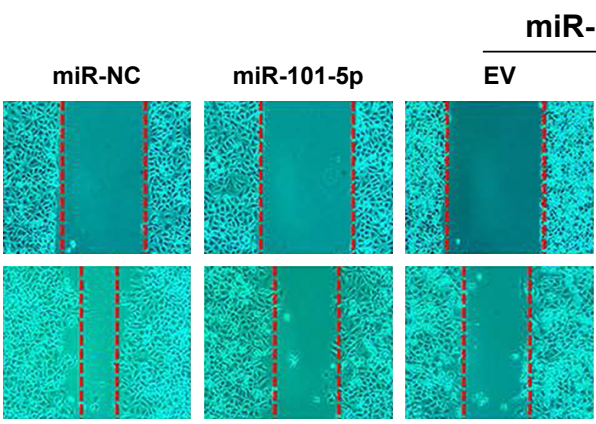

miR-101-5p

B

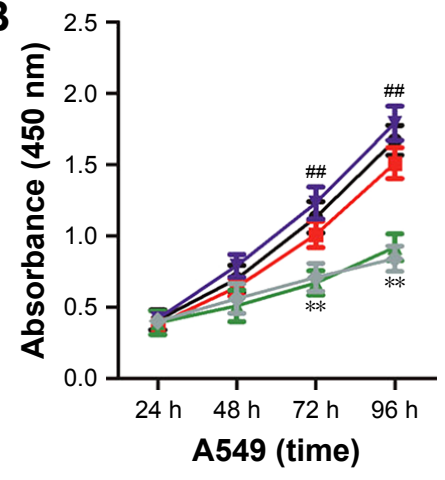

- Control

- $\operatorname{miR}-\mathrm{NC}$

- miR-101-5p

$\checkmark-\operatorname{miR}-101-5 p+E V$

$\rightarrow$ miR-101-5p + CXCL6
E

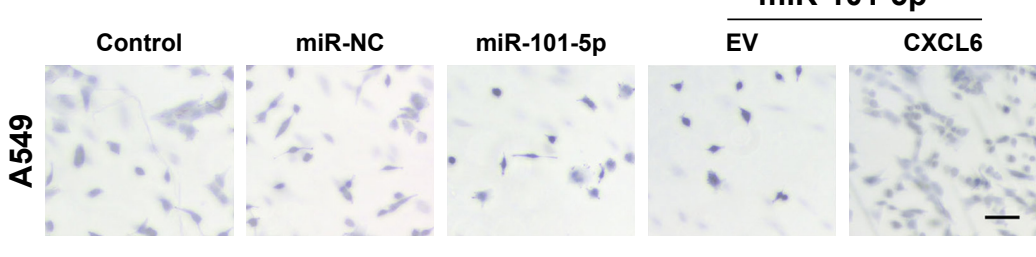

E

Figure 6 Overexpression of CXCL6 reverses the effect of miR-10I-5p in A549 cells.

Notes: (A) A549 cells that were transfected with miR-10I-5p and CXCL6 were subjected to immunofluorescence staining assay. (B) A549 cells were transfected with miR-10I-5p alone or miR-10I-5p in combination with CXCL6. The proliferation of A549 cells was detected using CCK-8 assay. (C) A549 cells were transfected with miR-I0I-5p alone or miR-I0I-5p in combination with CXCL6. Colony formation assay was conducted. (D) The migration of A549 cells was determined by wound healing assay. (E) A549 cells were transfected with miR-10I-5p alone or miR-10I-5p in combination with CXCL6. The invasion of A549 cells was measured by Transwell invasion assay. ${ }^{* * P}<0.01$ compared to control and $P<0.01$ compared to miR-10I-5p.

Abbreviations: CCK-8, cell counting kit-8; EV, empty vector; h, hours. 
A
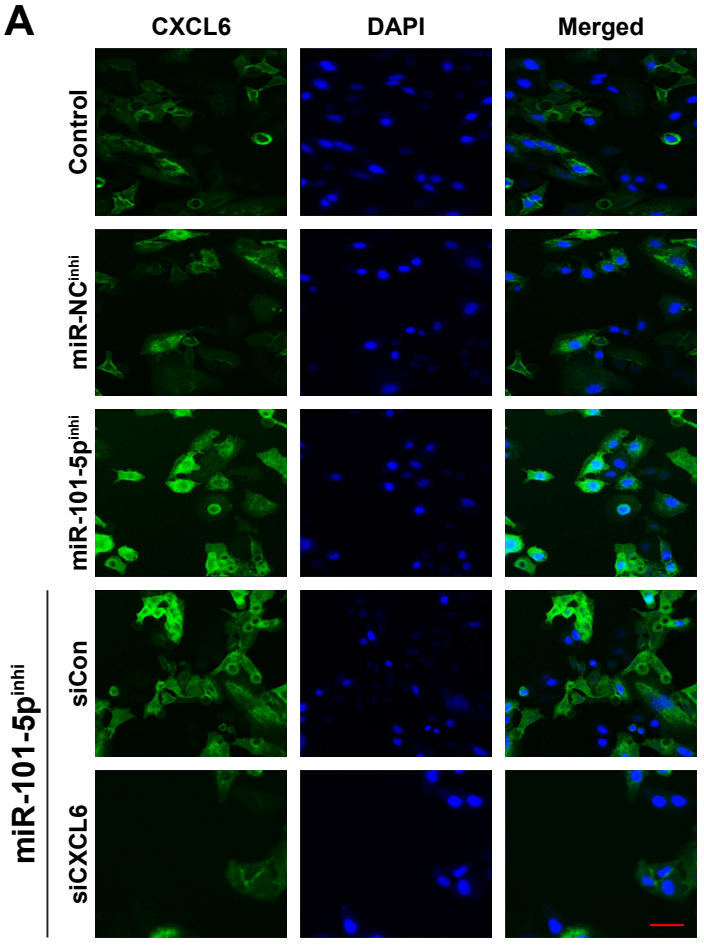

C
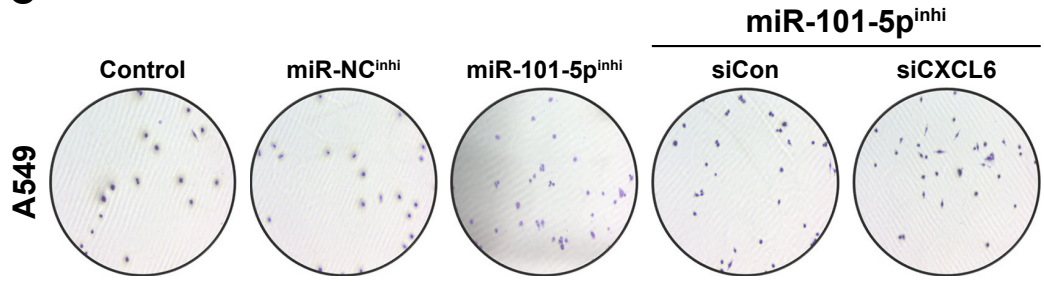

D

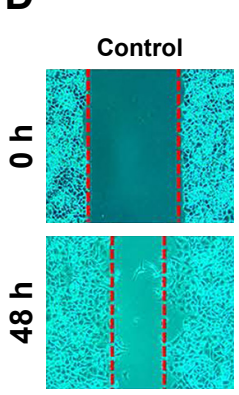

miR-NC inhi $^{\text {in }}$
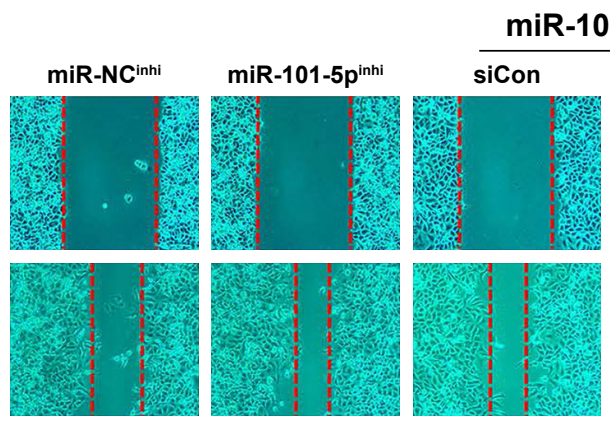

$p^{\text {inhi }}$

E

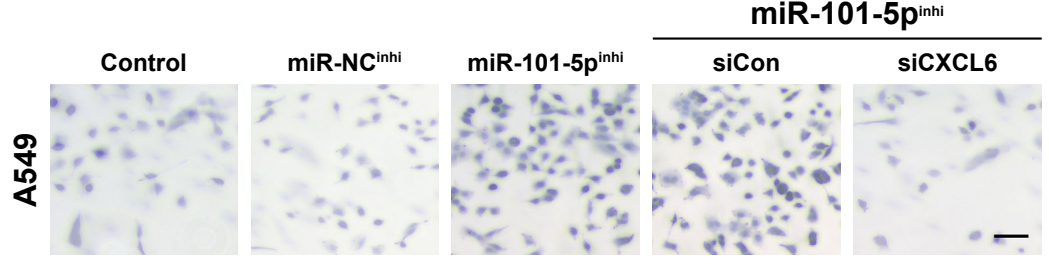

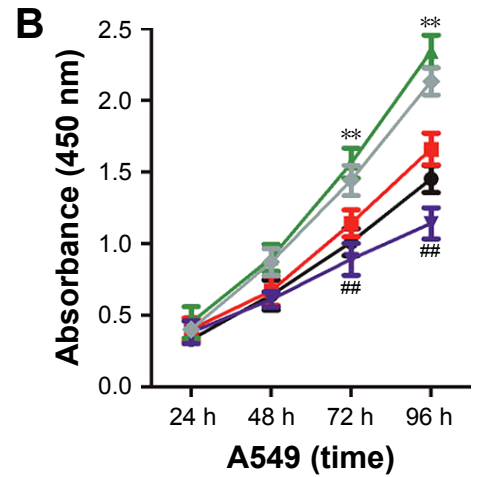

$\rightarrow$ Control
- miR-NCinhi
- miR-101-5p $p^{\text {inhi }}$
$\sim$ miR-101-5 $p^{\text {inhi }}+$ siCon
$\rightarrow$ miR-101-5p $p^{\text {inhi }}+$ siCXCL6
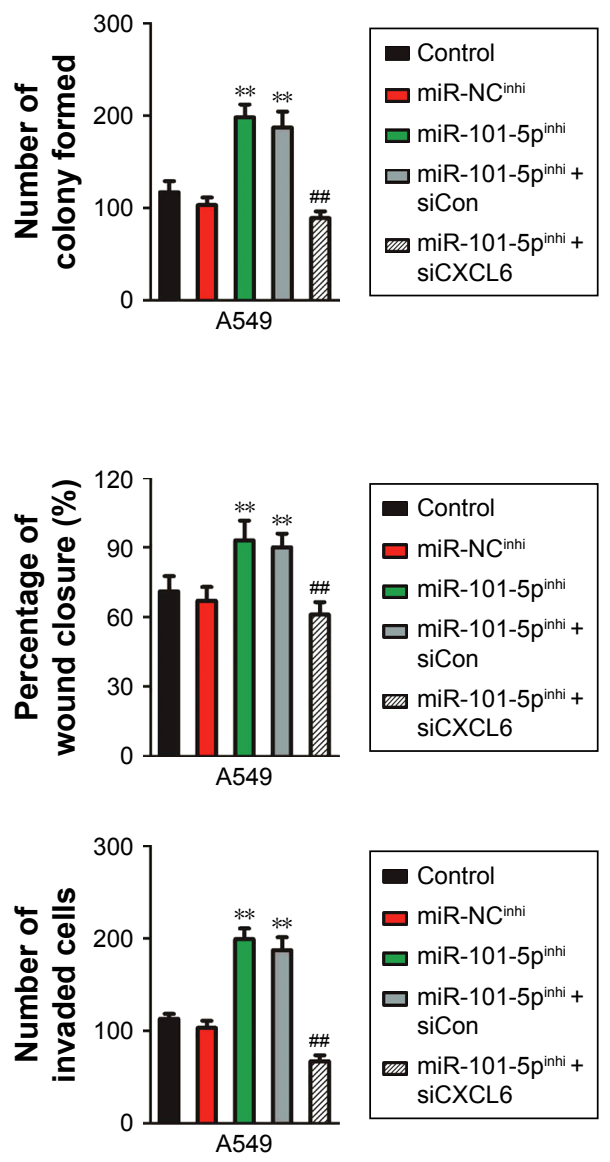

Figure 7 Downexpression of CXCL6 reverses the effect of miR-10I-5p in A549 cells.

Notes: (A) A549 cells that were transfected with miR-101-5 pinhi and siCXCL6 were subjected to immunofluorescence staining assay. (B) A549 cells were transfected with miR-10I-5 $p^{\text {inhi }}$ alone or miR-10I-5 $\mathrm{p}^{\text {inhi }}$ in combination with siCXCL6. The proliferation of A549 cells was detected using CCK-8 assay. (C) A549 cells were transfected with

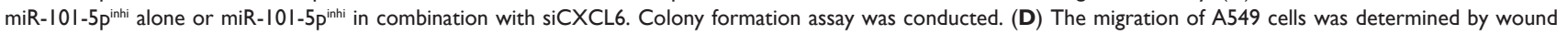
healing assay. (E) A549 cells were transfected with miR-10I-5p inhi alone or miR-10I-5p inhi in combination with siCXCL6. The invasion of A549 cells was analyzed by Transwell invasion assay. $* * P<0.01$ compared to control and ${ }^{\#} P<0.01$ compared to miR-10I-5 $p^{\text {inhi }}$.

Abbreviations: CCK-8, cell counting kit-8; h, hours; miR-I0I-5p inhi, miR-I0I-5p inhibitor. 
phenotypes of NSCLC cells. First, A549 cells were cotransfected with miR-101-5p and pcDNA3.1(+) that contain CXCL6. As expected, the suppressive roles of miR-101-5p in the growth, colony formation, migration and invasion of A549 cells were rescued by overexpression of CXCL6. A549 cells were also cotransfected with miR-101-5 $\mathrm{p}^{\text {inhi }}$ and siRNA targeting CXCL6. Downregulation of CXCL6 obviously inhibited the growth, migration and invasion of A549 cells that were promoted by miR-101-5 $\mathrm{p}^{\text {inhi }}$ transfection, suggesting that miR-101-5p exerted its functions by regulating the expression of CXCL6 in NSCLC.

\section{Conclusion}

We prove that miR-101-5p is underregulated in NSCLC and serves as a suppressive miRNA. Furthermore, upregulation of miR-101-5p inhibits the proliferation, migration and invasion of NSCLC cells in vitro as well as suppresses the metastasis and growth of NSCLC cells in vivo. In addition, we demonstrate that miR-101-5p regulates the growth and aggressiveness of NSCLC cells through regulating the expression of CXCL6. These results reveal the underlying mechanism by which miR-101-5p acts as a potential tumor suppressor in NSCLC.

\section{Disclosure}

The authors report no conflicts of interest in this work.

\section{References}

1. Shroff GS, Viswanathan C, Carter BW, et al. Staging lung cancer: metastasis. Radiol Clin North Am. 2018;56(3):411-418.

2. Legras A, Pécuchet N, Imbeaud S, et al. Epithelial-to-Mesenchymal transition and MicroRNAs in lung cancer. Cancers. 2017;9(8):101.

3. Pawlak K, Gabryel P, Kujawska A, et al. Long-term results of surgical treatment of non-small cell lung cancer in patients over 75 years of age. Kardiochir Torakochirurgia Pol. 2018;15(2):65-71.

4. Simonian M, Sharifi M, Nedaeinia R, et al. Evaluation of miR-21 inhibition and its impact on cancer susceptibility candidate 2 long noncoding RNA in colorectal cancer cell line. Adv Biomed Res. 2018; $7: 14$.

5. Xie WB, Liang LH, Wu KG, Kg W, et al. MiR-140 expression regulates cell proliferation and targets PD-L1 in NSCLC. Cell Physiol Biochem. 2018;46(2):654-663.

6. Zhang Y, Luo J, Wang X, et al. A comprehensive analysis of the predicted targets of miR-642b-3p associated with the long non-coding RNA HOXA11-AS in NSCLC cells. Oncol Lett. 2018;15(5):6147-6160.

7. Hua FF, Liu SS, Zhu LH, et al. MiRNA-338-3p regulates cervical cancer cells proliferation by targeting MACC1 through MAPK signaling pathway. Eur Rev Med Pharmacol Sci. 2017;21(23):5342-5352.

8. Cheung CC, Lun SW, Chung GT, et al. MicroRNA-183 suppresses cancer stem-like cell properties in EBV-associated nasopharyngeal carcinoma. BMC Cancer. 2016;16:495.

9. Bouyssou JM, Manier S, Huynh D, et al. Regulation of microRNAs in cancer metastasis. Biochim Biophys Acta. 2014;1845(2):255-265.

10. Reddy SD, Pakala SB, Ohshiro K, Rayala SK, Kumar R. MicroRNA-661, a c/EBPalpha target, inhibits metastatic tumor antigen 1 and regulates its functions. Cancer Res. 2009;69(14):5639-5642.
11. Zhang LY, Liu M, Li X, Tang H. miR-490-3p modulates cell growth and epithelial to mesenchymal transition of hepatocellular carcinoma cells by targeting endoplasmic reticulum-Golgi intermediate compartment protein 3 (ERGIC3). J Biol Chem. 2013;288(6):4035-4047.

12. Han L, Chen W, Xia Y, et al. MiR-101 inhibits the proliferation and metastasis of lung cancer by targeting zinc finger E-box binding homeobox 1. Am J Transl Res. 2018;10(4):1172-1183.

13. Wang L, Yao J, Sun H, et al. MicroRNA-101 suppresses progression of lung cancer through the PTEN/AKT signaling pathway by targeting DNA methyltransferase 3A. Oncol Lett. 2017;13(1):329-338.

14. Zhang X, He X, Liu Y, et al. MiR-101-3p inhibits the growth and metastasis of non-small cell lung cancer through blocking PI3K/AKT signal pathway by targeting MALAT-1. Biomed Pharmacother. 2017;93:1065-1073.

15. Karin N, Razon H. Chemokines beyond chemo-attraction: CXCL10 and its significant role in cancer and autoimmunity. Cytokine. 2018;109:24-28.

16. Karin N. Chemokines and cancer: new immune checkpoints for cancer therapy. Curr Opin Immunol. 2018;51:140-145.

17. Spaks A. Role of CXC group chemokines in lung cancer development and progression. J Thorac Dis. 2017;9(Suppl 3):S164-S171.

18. Bonecchi R, Locati M, Mantovani A. Chemokines and cancer: a fatal attraction. Cancer Cell. 2011;19(4):434-435.

19. Kruizinga RC, Bestebroer J, Berghuis P, et al. Role of chemokines and their receptors in cancer. Curr Pharm Des. 2009;15(29):3396-3416.

20. Li J, Tang Z, Wang H, et al. CXCL6 promotes non-small cell lung cancer cell survival and metastasis via down-regulation of miR-515-5p. Biomed Pharmacother. 2018;97:1182-1188.

21. Xu L, Duda DG, di Tomaso E, et al. Direct evidence that bevacizumab, an anti-VEGF antibody, up-regulates SDF1alpha, CXCR4, CXCL6, and neuropilin 1 in tumors from patients with rectal cancer. Cancer Res. 2009;69(20):7905-7910.

22. Zhu YM, Bagstaff SM, Woll PJ. Production and upregulation of granulocyte chemotactic protein-2/CXCL6 by IL-1beta and hypoxia in small cell lung cancer. Br J Cancer. 2006;94(12):1936-1941.

23. Ma JC, Sun XW, Su H, et al. Fibroblast-derived CXCL12/SDF-1 $\alpha$ promotes CXCL6 secretion and co-operatively enhances metastatic potential through the $\mathrm{PI} 3 \mathrm{~K} / \mathrm{Akt} / \mathrm{mTOR}$ pathway in colon cancer. World J Gastroenterol. 2017;23(28):5167-5178.

24. Verbeke H, Struyf S, Berghmans N, et al. Isotypic neutralizing antibodies against mouse GCP-2/CXCL6 inhibit melanoma growth and metastasis. Cancer Lett. 2011;302(1):54-62.

25. Gijsbers K, Gouwy M, Struyf S, et al. GCP-2/CXCL6 synergizes with other endothelial cell-derived chemokines in neutrophil mobilization and is associated with angiogenesis in gastrointestinal tumors. Exp Cell Res. 2005;303(2):331-342.

26. Gu S, Liang H, Qi D, et al. Knockdown of KIF26B inhibits breast cancer cell proliferation, migration, and invasion. Onco Targets Ther. 2018;11:3195-3203.

27. Zhao W, Geng D, Li S, Chen Z, Sun M. LncRNA HOTAIR influences cell growth, migration, invasion, and apoptosis via the miR-20a-5p/ HMGA2 axis in breast cancer. Cancer Med. 2018;7(3):842-855.

28. Zhang J, Li Y. Oncogenic miR-155 down-regulated upon activation of antitumor cytotoxic T lymphocytes by the fusion of dendritic cells with breast carcinoma cells. Eur Rev Med Pharmacol Sci. 2017;21(5): 1027-1031.

29. Zhang S, Ge W, Zou G, et al. MiR-382 targets GOLM1 to inhibit metastasis of hepatocellular carcinoma and its down-regulation predicts a poor survival. Am J Cancer Res. 2018;8(1):120-131.

30. Zang Y, Wang T, Pan J, Gao F. miR-215 promotes cell migration and invasion of gastric cancer cell lines by targeting FOXO1. Neoplasma. 2017;64(4):579-587.

31. Fan X, Zhou S, Zheng M, et al. MiR-199a-3p enhances breast cancer cell sensitivity to cisplatin by downregulating TFAM (TFAM). Biomed Pharmacother. 2017;88:507-514.

32. Vychytilova-Faltejskova P, Merhautova J, Machackova T, et al. MiR-215-5p is a tumor suppressor in colorectal cancer targeting EGFR ligand epiregulin and its transcriptional inducer HOXB9. Oncogenesis. 2017;6(11):399. 
33. Wei C, Zhang X, He S, et al. MicroRNA-219-5p inhibits the proliferation, migration, and invasion of epithelial ovarian cancer cells by targeting the Twist/Wnt/ $\beta$-catenin signaling pathway. Gene. 2017;637: 25-32.

34. Zheng HB, Zheng XG, Liu BP. miRNA-101 inhibits ovarian cancer cells proliferation and invasion by down-regulating expression of SOCS-2. Int J Clin Exp Med. 2015;8(11):20263-20270.
35. Yang X, Pang YY, He RQ, RqH, et al. Diagnostic value of strand-specific miRNA-101-3p and miRNA-101-5p for hepatocellular carcinoma and a bioinformatic analysis of their possible mechanism of action. FEBS Open Bio. 2018;8(1):64-84. 


\section{Supplementary materials}

Table SI Clinical characteristics of 55 test samples obtained from patients with NSCLC

\begin{tabular}{|c|c|c|c|c|c|}
\hline Characteristics & AC & SCC & LCC & LCNEC & Total \\
\hline Number of cases & 31 & 16 & 7 & I & 55 \\
\hline Median age (range), years & $65(36-83)$ & 71 (56-83) & $59(5 I-79)$ & 76 & $68(36-83)$ \\
\hline \multicolumn{6}{|l|}{ Gender } \\
\hline Male & 21 & 12 & 6 & 1 & 40 \\
\hline Female & 10 & 4 & 1 & 0 & 15 \\
\hline \multicolumn{6}{|l|}{ Smoking } \\
\hline Ever & 23 & 11 & 5 & I & 40 \\
\hline Never & 7 & 3 & 2 & 0 & 12 \\
\hline NA & 1 & 2 & 0 & 0 & 3 \\
\hline \multicolumn{6}{|l|}{ Stage } \\
\hline I & 19 & 9 & 6 & I & 35 \\
\hline$\|$ & 7 & 4 & 1 & 0 & 12 \\
\hline III & 3 & 2 & 0 & 0 & 5 \\
\hline IV & 1 & 1 & 0 & 0 & 2 \\
\hline NA & I & 0 & 0 & 0 & I \\
\hline
\end{tabular}

Abbreviations: AC, adenocarcinoma; LCC, large-cell lung carcinoma; LCNEC, large-cell neuroendocrine carcinoma; NA, data not available; NSCLC, non-small-cell lung carcinoma; SCC, squamous cell carcinoma.

Table S2 Results of EGFR and KRAS mutation status

\begin{tabular}{l|l}
\hline & $\mathbf{N}=\mathbf{5 5}$ \\
\hline EGFR gene & \\
Mutation & 22 \\
Exon I8 (G7I9X) & 7 \\
Exon 19 (19del) & 5 \\
Exon 2I & 3 \\
L858R & 1 \\
L86IQ & 2 \\
Exon 20 & 6 \\
T790M & 2 \\
H337_V774ins H & 4 \\
S768I & 0 \\
Combination of two mutations & 1 \\
I9del+20T790M & 1 \\
Wild type & 33 \\
KRAS gene & \\
Mutation & 17 \\
Exon 2 & 11 \\
Exon 3 & 6 \\
Wild type & 38 \\
Combination of EGFR and KRAS mutations & 2 \\
\hline
\end{tabular}

Table S3 Candidate reference genes for normalization and the expression stability were calculated by the NormFinder program

\begin{tabular}{l|l}
\hline Gene name & Stability value \\
\hline GAPDH & 0.214 \\
U6 & 0.126 \\
ACTB & 0.649 \\
RPL32 & 0.509 \\
RPLI3A & 0.837 \\
HPRTI & 0.936 \\
\hline
\end{tabular}

OncoTargets and Therapy

\section{Publish your work in this journal}

OncoTargets and Therapy is an international, peer-reviewed, open access journal focusing on the pathological basis of all cancers, potential targets for therapy and treatment protocols employed to improve the management of cancer patients. The journal also focuses on the impact of management programs and new therapeutic agents and protocols on

\section{Dovepress}

patient perspectives such as quality of life, adherence and satisfaction. The manuscript management system is completely online and includes a very quick and fair peer-review system, which is all easy to use. Visit http://www.dovepress.com/testimonials.php to read real quotes from published authors. 